\title{
Le fakamisimisi de Futuna ou l'esthétique du travail quotidien
}

\section{Adriano Favole}

\section{Q OpenEdition}

1 Journals

Édition électronique

URL : http://journals.openedition.org/jso/593

DOI : $10.4000 /$ jso. 593

ISSN : $1760-7256$

Éditeur

Société des océanistes

Édition imprimée

Date de publication : 1 décembre 2006

Pagination : 123-139

ISSN : 0300-953x

\section{Référence électronique}

Adriano Favole, "Le fakamisimisi de Futuna ou l'esthétique du travail quotidien », Journal de la Société des Océanistes [En ligne], 122-123 | Année 2006, mis en ligne le 01 décembre 2009, consulté le 01 mai 2019. URL : http://journals.openedition.org/jso/593 ; DOI : 10.4000/jso.593

\section{() Tous droits réservés}




\title{
Le fakamisimisi de Futuna ou l'esthétique du travail quotidien ${ }^{1}$
}

\author{
par
}

Adriano FAVOLE*

\section{RÉSUMÉ}

Le but de cet article est d'examiner un genre inédit de la riche et vivante littérature orale futunienne : le fakamisimisi. Déclamé au cours des cérémonies redistributives appelées katoaga, le fakamisimisi est un texte qui présente de grandes difficultés de traduction et d'interprétation. À la différence des fakamatala, des miō et d'autres genres de la littérature orale mieux connus, le fakamisimisi ne parle pas du passé pré-chrétien, de héros (to'a), de chefs (aliki) ou de divinités (atua). Il s'intéresse plutôt au travail quotidien des hommes et des femmes et aux produits de leur fatigue : les plantations de kava, d'ignames et de taros, l'élevage de cochons, etc., auxquels les différentes strophes sont dédiées. Récité par un aliki devant le mala'e du village recouvert de " biens » et de " richesses », le fakamisimisi se veut être une exaltation des produits du travail humain et des techniques permettant de les obtenir. À partir de la traduction et de l'interprétation d'un fakamisimisi composé et déclamé en 1997 à l'occasion d'un katoaga dans le village d'Ono (chefferie d'Alo), l'auteur réfléchit ici à la dimension esthétique (ou ethnoesthétique) des productions et des rites qui les célèbrent. Il avance la thèse selon laquelle l'importance de certains aspects de la coutume - tels les rites katoaga dans la société futunienne d'aujourd'hui - est à rechercher non seulement dans les " politiques de la tradition" et dans leur capacité de redistribuer les ressources et d'éviter l'apparition d'importantes inégalités sociales, mais aussi dans leur dimension esthétique et dans leur capacité de mise en valeur des techniques de production et des produits locaux vis-à-vis des marchandises du marché mondialisé.

Mots-CLÉs : Futuna, tradition orale, fête, esthétique du travail.

\section{ABSTRACT}

The aim of this article is to analyse a text of the rich and lively oral tradition of East Futuna, which has never been the object of an anthropological or linguistic study: the fakamisimisi.

This text is pronounced by a chief in the village plaza (mala'e) where local «richness» is displayed during the redistributive ceremonies named katoaga. Unlike miō, fakamatala and other kinds of Futunan oral performances, the fakamisimisi is not dealing with heroes (to'a), chiefs (aliki) or divinities (atua) of the pre-christian past. It deals with the everyday work of men and women and with the products of this work: kava, yam, taro, pigs etc., which are the central topics of the different stanzas of the poem.

Starting out with the translation and interpretation of a fakamisimisi performed in October 1997 during a katoaga in Ono village (chiefdom of Alo), this article reflects upon the aesthetic (or ethno-aesthetic) dimension of human production and of Polynesian redistributive rites. It shows that one has to understand the importance of particular aspects of the «kustom»-such as the contemporary katoaga rites in Futuna - not only in the light of the "politics of tradition», or as an efforts to avoid the spread of social and economic inequalities, but as a mean to stress the value of local products and processes against the standardized goods and activities of the globalised market.

KEYwords: East Futuna, oral tradition, work, ethnoaesthetic.

1. Mes recherches sur le terrain ont été effectuées à Futuna pendant environ sept mois entre 1996 et 1997. Puis, en 1999 et en 2004, durant deux courts séjours en Nouvelle-Calédonie, j'ai eu l'occasion de m'occuper de la communauté de Futuniens qui vivent sur la Grande Terre. En plus de Soane Masei qui, comme je le mentionne plus loin, a été le principal auteur de la traduction et de l'interprétation du texte faisant l'objet de cette étude, je remercie Petelo Leleivai, Koleta Filitika Cristini et Claire Moyse pour leur révision et leurs précieux commentaires. Je remercie Pier Paolo Viazzo et Silvia Forni pour avoir lu et commenté la version italienne de l'article.

* Maître de conférences à l'université de Turin, Département de Sciences anthropologiques, archéologiques et historicoterritoriales, favole.bessone@isiline.it 
La publication, en 1995, du majestueux recueil de textes Ko le fonu tu'a limulimua, $L a$ tortue au dos moussu (Frimigacci et al., 1995) a révélé la richesse et la vivacité persistante de la littérature orale futunienne. Les fakamatala, les fanaga, les miō et les chants qui accompagnent les danses takofe et tapaki, recueillis au cours des années quatre-vingts et réunis dans ce volume, représentent aujourd'hui encore un savoir répandu et transmis sur l'île. Ces textes, qui renferment des événements et des interprétations de l'histoire et se distinguent par leur richesse et la variété de leur forme littéraire, exercent aussi un rôle à la fois " pragmatique » et " performatif ». Comme je l'ai déjà mentionné ailleurs (Favole, 2000a), les frontières entre les différentes partitions territoriales de l'île ( $k a \bar{a} i g a^{2}$, villages, royaumes) sont contenues dans les textes de la tradition orale - qui s'apparentent, en définitive, à un cadastre - et c'est au sein de ces mêmes textes que les chefs porteurs de titres (aliki) trouvent la source primaire de leur autorité et de leur prestige. Ces récits, qui se présentent tantôt comme des « documents historiques » (les fakamatala), tantôt comme des « contes » ou des « histoires » à finalité didactique (les fanaga), tracent une sorte de géographie historico-mythologique en transformant l'environnement naturel en paysage culturel : les roches, les précipices et les fissures du récif deviennent les signes d'un passé peuplé de divinités (atua, temonio) et d'ancêtres-héros (to'a).

L'aspect « performatif » de certains genres de la littérature orale futunienne est lié au fait qu'ils sont récités ou déclamés lors de cérémonies telles que les kava, les katoaga (fêtes de distribution de vivres) et les festivals de danses (kelemesi). Comme l'a écrit Patrick Kirch, auteur d'une recherche approfondie de nature ethnoarchéologique dans les années soixante-dix à Futuna, la récitation des miō et les danses takofe et tapaki (accompagnées de leurs chants respectifs) constituent l'un des aspects centraux d'un katoaga:

" Miō in particular celebrate heroic acts of former chiefs and warriors [ \&] Tapaki and takofe are dances accompanied by poetic chants that likewise glorify past culture heroes. » (Kirch, 1994a : 143)

Cette connexion entre la tradition orale et le passé préchrétien est également mise en évidence par les éditeurs de La tortue au dos moussu:

« La tradition véhiculée par les fakamatala se présente d'abord comme la chronique des faits et gestes des héros fondateurs avec cette particularité de conjoindre la temporalité avec l'espace où se sont déroulés les événements. » (Frimigacci et al., 1995 : 15)

Elle contribue peut-être à expliquer les raisons d'une absence importante. En effet, un genre de la tradition orale présentant, selon moi, plusieurs aspects dignes d'intérêt, ne trouve pas encore sa place dans les recueils publiés jusqu'à présent : il s'agit du fakamisimisi qui est déclamé lors des katoaga. À la différence des miō et des fakamatala, le fakamisimisi n'a pas l'allure de la " grande histoire ». Il se présente plutôt comme une célébration du travail quotidien des gens dans les jardins.

\section{Texte et contexte: le fakamisimisi, moment important d'un katoaga}

Le dictionnaire du père Isidore Grézel ne considérait pas le fakamisimisi comme un genre de la littérature orale. Le missionnaire proposait toutefois des significations de ce terme qui contribuent bien à définir la nature du texte. Fakamisimisi est selon lui :

« Applaudir, louer, complimenter, flatter ; pousser à..., exciter à... » (Grézel, 1878 : 103)

Claire Moyse (1993 : 105), dans son dictionnaire édité plus d'un siècle après, rapporte ce terme à la définition suivante :

«Discours de remerciement émis lors des fêtes de distribution de vivres. »

Il nous faut donc partir précisément de ces « fêtes de distribution de vivres », appelées katoaga, pour situer le fakamisimisi dans le contexte pour lequel il est composé et lors duquel il est déclamé de façon solennelle.

La scène ethnographique dans le cadre de laquelle j'ai recueilli la version du fakamisimisi présenté en appendice est un katoaga célébré le $1^{\text {er }}$ octobre 1997 dans le village d'Ono (chefferie d'Alo), à l'occasion de la fête de Sainte-Thérèse, la patronne du village. Bien que l'hypothèse d'organiser ce katoaga ait été prise dès l'année précédente, la décision est officialisée lors du fono lasi, la "grande assemblée » du royaume réunie le dimanche 14 septembre à laquelle participent le délégué du préfet (l'autorité territoriale la plus importante de l'île), le « roi » Tu'i Agaifo et les cinq "grands chefs 》 (aliki lasi) d'Alo ${ }^{3}$. Le jour même, en fin de matinée, le fono

2. Le mot käiga désigne en même temps les différentes parcelles de terre qui composent un village et les groupes de parenté qui $\mathrm{y}$ résident.

3. Il s'agit, dans l'ordre hiérarchique établi par le rituel du kava, du Tiafoi, Sa'atula, Tu'i Asoa, Tu'i Sa'avaka et Vakalasi. 
fakafenua, l'assemblée du village d'Ono, présidée par le Tu'i Asoa et par deux «petits chefs" (Ma'uifa et Fainuvele), prend d'importantes décisions quant à la forme que le katoaga doit revêtir. L'assemblée choisit de célébrer la fête selon la modalité appelée tagata-fatogia plutôt que selon celle portant le nom de fai'umu. Le tagata-fatogia désigne un katoaga au cours duquel chaque homme adulte (tagata) qui s'acquitte du fatogia (voir infra) envers le chef du village se doit d'offrir au moins un panier de nourriture et un cochon. La forme fai'umu du katoaga, dans laquelle le four ('umu) et non pas l'homme-fatogia constitue l'unité de référence, est écartée. Dans la forme fai'umu, le katoaga aurait été moins important puisque le nombre de fours dans un kāiga est toujours inférieur au nombre de tagata-fatogia ${ }^{4}$.

«Cette année - annonce à cette occasion le Tu’i Asoa - marque le $150^{\mathrm{e}}$ anniversaire de la mort de la sainte patronne, il faut donc célébrer un grand katoaga. »

La même assemblée décide alors du type de nourriture qui doit remplir les paniers. Après une discussion animée, le choix se porte sur le ta'o tākisi, un aliment obtenu en plongeant du taro et des fruits cuits de l'arbre à pain (mei) dans du lait de coco. Le don d'un panier de ta'o tākisi s'accompagne bien évidemment - le sujet n'est d'ailleurs pas même abordé durant l'assemblée en raison de son évidence pour chacun des participants! - d'un cochon de dimension plus ou moins grande en fonction des ambitions et des possibilités économiques de chaque donateur. Les décisions du fono lasi et du fono fakafenua sont communiquées par les deux petits chefs du village au fono fafine, l'assemblée féminine. Les chefs disent aux femmes qu'elles doivent s'occuper de la préparation des petits déjeuners pour le matin de la fête. Certaines d'entre elles réclament d'exercer un rôle plus important dans le cadre du katoaga, mais en vain. Toutefois, les jours suivants, les femmes font pression sur la chefferie en dénonçant l'« avarice » du Tu'i Asoa, c'est-àdire sa volonté d' "économiser» : elles demandent alors à organiser un second katoaga dans l'après-midi. Chaque femme-fatogia fait au moins don d'un gasue 5 . En outre, elles préparent une danse visant à recueillir des fonds pour les coopératives de village et pour la mission catholique de Kolopelu. La pression est si forte que le
Tu'i Asoa risque d'être détrôné et se voit contraint de se soumettre aux requêtes féminines. Quant aux questions relatives à la coutume, l'opposition des femmes face à la domination masculine s'exprime souvent, à Futuna, à travers le désir d'une plus grande « visibilité » et d'une participation aux rituels.

Mon attention s'est focalisée sur les détails de la préparation du katoaga car ils contribuent à l'explication des contenus du texte fakamisimisi. La grandeur d'un katoaga traduit le bien-être et la prospérité de l'île et révèle l'adresse et la force des hommes et des femmes du village organisateur concernant tout d'abord le travail laborieux de la terre. Ensuite, comme un essai de vulgarisation - renfermant toutefois une documentation ethnographique précieuse - l'a déjà soulignée (Di Piazza et al., 1991), la préparation, la cuisson et la consommation de la nourriture à Futuna présentent un caractère "esthétique» marqué. Au cours du texte fakamisimisi, nous trouvons précisément, d'une part, une exaltation du travail dans les champs et, d'autre part, la célébration de la beauté des produits qui en découlent et qui sont exposés solennellement sur la place (mala'e) du village lors des katoaga, pour être ensuite redistribués.

Le concept de fatogia est ici extrêmement important. Défini en général comme un ensemble d'« obligations coutumières" et, dans sa forme verbale, par le fait « d'être obligé, d'avoir des obligations coutumières » (Moyse, 1993: 136), le concept de fatogia recouvre, selon moi, un champ sémantique bien plus vaste. À Futuna, l'exercice du fatogia est lié au passage à l'âge adulte. Pour les hommes, il est associé, de manière significative, à la circoncision ${ }^{6}$. Lorsqu'un parent considère que son fils ou sa fille sont prêts à "être » fatogia, il communique sa décision au chef du village. Le fatogia se rapporte au käiga d'appartenance : " on fait» le fatogia pour un kāiga spécifique et puisque ces partitions territoriales (qui coïncident avec des regroupements de parenté exogames) sont soumises à l'autorité d'un chef porteur de titre, il en résulte que chaque homme et chaque femme-fatogia dépendent d'un chef précis.

Être hommes ou femmes-fatogia entraîne la possibilité de participer aux réunions du village (fono) et aux travaux d'intérêt collectif. Les hommes et les femmes-fatogia ont non seulement le droit et le devoir de travailler la terre, de pêcher,

4. À propos de l'organisation de la parenté à Futuna, se référer à Michel Panoff (1970), Françoise Douaire-Marsaudon (1998) et Adriano Favole (2000a).

5. «Ensemble de vivres, transportés dans des paniers ga'ati, pour les fêtes de distribution de vivres » (Moyse, 1993 : 174).

6. Autrefois partie intégrante du cycle rituel annuel (Kirch, 1994b), la circoncision a lieu aujourd'hui dans le petit dispensaire de l'île. 
d'élever des cochons ou d'avoir un travail salarié, mais ils peuvent également se rendre aux terrains communs du village pour y cultiver une parcelle de terre ou, avec l'autorisation du chef, y construire des fale (maisons traditionnelles). En outre, ils peuvent se servir d'instruments de travail collectifs (bateaux, camions, etc.) en adhérant à l'une des coopératives de village. Le fatogia comporte le droit et le devoir de faire don de nourriture, de cochons ou d'argent pour un katoaga, de participer activement aux festivals de danse (kelemesi) et, pour les hommes, de participer aux assemblées du soir (tanasu) pour discuter (lakulaku pati) et boire du kava. Bien que de nombreux habitants de l'île mentionnent souvent les aspects coercitifs et «lourds » du fatogia, il ne s'agit pas seulement d'un ensemble d' « obligations». Être homme ou femmefatogia signifie faire partie, de plein droit, de la société et "participer» à la vie sociale. Par conséquent, il me semble utile de traduire le terme fatogia par le terme « citoyenneté ». Les tagata-fatogia et les fafine-fatogia sont les " citoyens » futuniens investis de droits et de devoirs, en vertu de leur affiliation parentale, territoriale (le kāiga) et politique (l'aliki pour lequel « on fait» le fatogia). Le texte fakamisimisi peut être conçu non seulement comme un discours de remerciement pour le travail, la beauté et l'abondance des produits offerts et exposés sur le mala'e, mais aussi comme une célébration de la "citoyenneté », de l'appartenance à une collectivité sociale avec les droits et les responsabilités - notamment celles liées au travail - qu'elle comporte.

Dans la mesure où les sources d'archives ainsi que les textes ethnographiques se réfèrent très souvent aux katoaga futuniens ${ }^{7}$, je n'en propose pas ici une description détaillée, me limitant à évoquer certains aspects de la cérémonie du $1^{\text {er }}$ octobre 1997 dans le but de contextualiser le texte fakamisimisi faisant l'objet de notre étude. La nuit précédant le katoaga est frénétique et intense. Près de chaque fale se trouvent des cochons de taille plus ou moins importante ainsi que des tas de feuilles d'arbres à pain qui serviront à gonfler leur ventre pour les faire paraître plus gros. Les paniers de mei et de taros s'amoncellent çà et là. À l'intérieur des fale, contenues dans de gigantesques feuilles de kape, des mil- liers de fleurs de tiale, d'orchidée, de jasmin et d'hibiscus accompagnées de fruits parfumés attendent d'être transformées en colliers. Vers minuit, les coups de feu des fusils, les coups de bâton et les cris des cochons marquent le début des travaux. Peu de temps après, les fours commencent à brûler : tout le village est enveloppé d'une lumière irréelle qui prend corps en filtrant à travers la fumée épaisse qui s'élève des monceaux enflammés. Un parfum intense de feuilles fanées envahit les maisons et les cabanes. Divisés en groupes dans les différents kāiga, les hommes s'occupent des cochons et des ta'o tākisi tandis que les femmes préparent des colliers de fleurs.

Peu avant l'aube, la grande esplanade publique du village est parcourue par un va-et-vient incessant de voitures chargées de cochons et de paniers de nourriture. Les cochons les plus gros, cuits dans le four, les ventres gonflés de façon anormale par les feuilles, sont peints avec le rouge du curcuma (ama). En grande hâte et sous la direction des anciens, les jeunes disposent les « richesses » sur la place. Le premier rang, près $\mathrm{du}$ fale au sein duquel prend place toute la chefferie, est occupé par des pieds de kava. Au deuxième rang trônent d'énormes cochons peints en rouge déposés sur des sacs de riz et d'étoffes d'écorce (siapo) suivis de cochons rouges de dimension plus réduite, placés sur des paniers de ta'o tākisi. Enfin, des petits cochons noirs sont disposés sur des paniers de ta'o tākisi tandis que des paniers de nourriture « traditionnelle » sont accompagnés de produits occidentaux comme des conserves de viande. Près de la plage, au-delà de la route côtière, s'élèvent cinq 'umu mata, des « colonnes » de nourritures crues (ignames, taros, fruits de l'arbre à pain, sacs de riz) surmontées de cochons et de siapo, offertes par un conseiller territorial du village d'Ono et par les quatre grands chefs des autres villages $\mathrm{d}^{\prime} \mathrm{Alo}^{8}$. Ce tableau des « richesses » est complété par un étendard formé d'une centaine de pièces d'étoffe colorées (manu). Le spectacle de plus de cent cochons et de l'ensemble des " richesses » éclairées par les premiers rayons du soleil est de toute beauté. C'est ce même spectacle que le compositeur du fakamisimisi se charge de traduire en vers.

Le déroulement de la fête prévoit tout d'abord une messe célébrée dans la nouvelle cathédrale

7. Sans prétention d'exhaustivité, nous pouvons citer, pour les sources plus anciennes, le journal des Hollandais Jacob Le Maire et Willem Schouten, premiers Européens à débarquer sur l'île (de Brosse, 1756 : 386-388); le Journal du père Pierre Chanel (1960 passim) ; Les Écrits du père Louis C. Servant $(1996: 164)$; certains documents d'archives auxquels se réfère Frédéric Angleviel dans son livre consacré à l'histoire des missions maristes à Wallis-et-Futuna (1994:111). Quant aux sources ethnographiques du vingtième siècle, citons notamment Edwin Burrows (1936 : 200-205); Daniel Frimigacci (1990 passim); Patrick Kirch (1994a : 199-204); Dominique Pechberty (1998) et Adriano Favole (2000a, 2000b).

8. Les katoaga peuvent être qualifiés de rites d'échange cérémoniel compétitifs. Donner des « richesses » pour un katoaga est très important pour quelqu'un qui aspire à entrer dans l'arène politique. 
d'Ono. Après un petit déjeuner copieux a lieu le premier des deux rituels kava devant le Tu'i Agaifo, tous les aliki du royaume et les autorités territoriales. Au terme de ce rituel, le récipient pour le kava (tāno'a) est déplacé en dehors du fale pour le second kava appelé kava fafo (kava externe). Tous les hommes-fatogia du village participent à ce kava assis derrière le tāno'a, placé près de la route à quelques dizaines de mètres du fale dans lequel les chefs inversent leur position habituelle en tournant leur visage vers la mer ( $k i$ tai). Le kava fafo est célébré pour tout le kakai, le «peuple » des chefs : la célébration à l'extérieur permet, en effet, une participation chorale. Les femmes qui-comme nous le savons - ne participent pas au kava, ont toutefois la possibilité de suivre de près le rituel, assises en marge de la place. C'est lors de cette phase du katoaga que, avant la récitation d'un miō et la distribution du kava, le fakamisimisi est déclamé avec autorité par le grand chef du village de Mala'e. La distribution des biens exposés sur la place, précédée d'une courte oraison du prêtre catholique, conclut la longue matinée rituelle. L'après-midi, la danse, animée par les femmes d'Ono, ainsi qu'un second katoaga féminin, mettent fin à cette journée de fête.

\section{Composition, déclamation et traduction du texte}

La recherche anthropologique est souvent le fruit de rencontres imprévisibles et d'événements inattendus au cours desquels le hasard joue un rôle considérable. Même si je n'avais pas pensé consacrer une partie importante de ma recherche sur le terrain à l'analyse de textes de la tradition orale, le fakamisimisi a attiré mon attention le jour où, vers la fin du mois de septembre 1997, mon hôte Setefano Takaniko me demande de lui prêter, quelques jours durant, mon enregistreur portable. Setefano Takaniko - conseiller territorial, responsable du Service culturel de Futunaest également devenu, depuis quelque temps, le grand chef (aliki lasi) du village de Mala'e, où réside d'ailleurs sa famille d'origine, portant ainsi le titre de $\mathrm{Sa}{ }^{\text {atula }}{ }^{9}$. Quelques jours après la cérémonie d'investiture, il commence à fréquenter intensément les anciens du royaume. Le Sa'atula s'informe (dans le sens d'" être formé à...») sur sa nouvelle activité d'aliki auprès de ceux qui sont considérés comme étant les dépositaires du savoir traditionnel. L'apprentissage des textes de la littérature orale constitue un devoir indispensable pour un chef futunien. En vue du katoaga du $1^{\text {er }}$ octobre 1997, le nouveau chef a décidé de composer et de déclamer un fakamisimisi pour le village d'Ono. Par convention et par « politesse », il est estimé que, lors de ces occasions, un chef extérieur au village organisateur doit se charger de prononcer le discours de remerciement. Afin de mieux mémoriser le texte qu'il compose peu à peu mentalement, le Sa'atula me demande de lui prêter l'enregistreur tout en me laissant, par la suite, la copie enregistrée du texte qu'il a préparé. Le texte publié en appendice est donc celui que le Sa'atula a ainsi enregistré. Du point de vue terminologique, celle faite durant la performance du $1^{\text {er }}$ octobre présente d'ailleurs quelques rares variations insignifiantes ${ }^{10}$. Les différences sont, en revanche, considérables de par le ton et l'emphase avec lesquels est prononcé le fakamisimisi dans le contexte rituel : le Sa'atula se met debout, parcourt la place du village à partir du fale et, en se déplaçant lentement vers la mer, s'arrête devant chacune des rangées de biens afin de remercier les chefs et le peuple pour l'abondance et la beauté des produits exposés. La voix est forte et solennelle, le visage continuellement en mouvement pour s'adresser aux différents groupes de l'auditoire (les chefs dans le fale, les hommes derrière le tāno' $a$, les femmes assises autour de la place) et il fait de longues pauses au terme de chaque strophe: la performance du chef de Mala'e est saluée par de longs applaudissements retentissants.

Dès le départ, la traduction du texte se révèle complexe. Bien que mes jeunes collaborateurs habituels connaissent très bien l'objectif du fakamisimisi (remercier les hommes et les femmes pour leur travail et pour les biens offerts et exposés) et, à grands traits, son contenu (une série de strophes "monographiques » consacrées à chacun des produits), ils montrent de grandes difficultés à traduire le texte. Les anciens avec lesquels je discute les principaux thèmes de ma recherche (centrée sur l'organisation politique) ne m'apportent pas une grande aide. Ils me disent d'aller voir plutôt dans les miō et surtout dans les fakamatala où je trouverai des informations biens plus importantes sur la coutume et l'histoire (la " grande histoire ») futunienne ! À leurs yeux, le fakamisimisi apparaît peut-être un genre trop « humble », qui ne doit pas être particulièrement intéressant pour un chercheur étranger qui s'occupe de politique locale. La traduction que je présente en appendice doit être

9. NDLR. « Titre à Alo (grand chef de Mala'e) et à Sigave (grand chef de Vaisei)» (Moyse-Faurie, $1993: 335$ ).

10. J'ai enregistré également cette performance, non sans difficulté, étant donné que je ne pouvais pas me lever pour suivre le chef dans sa performance. 


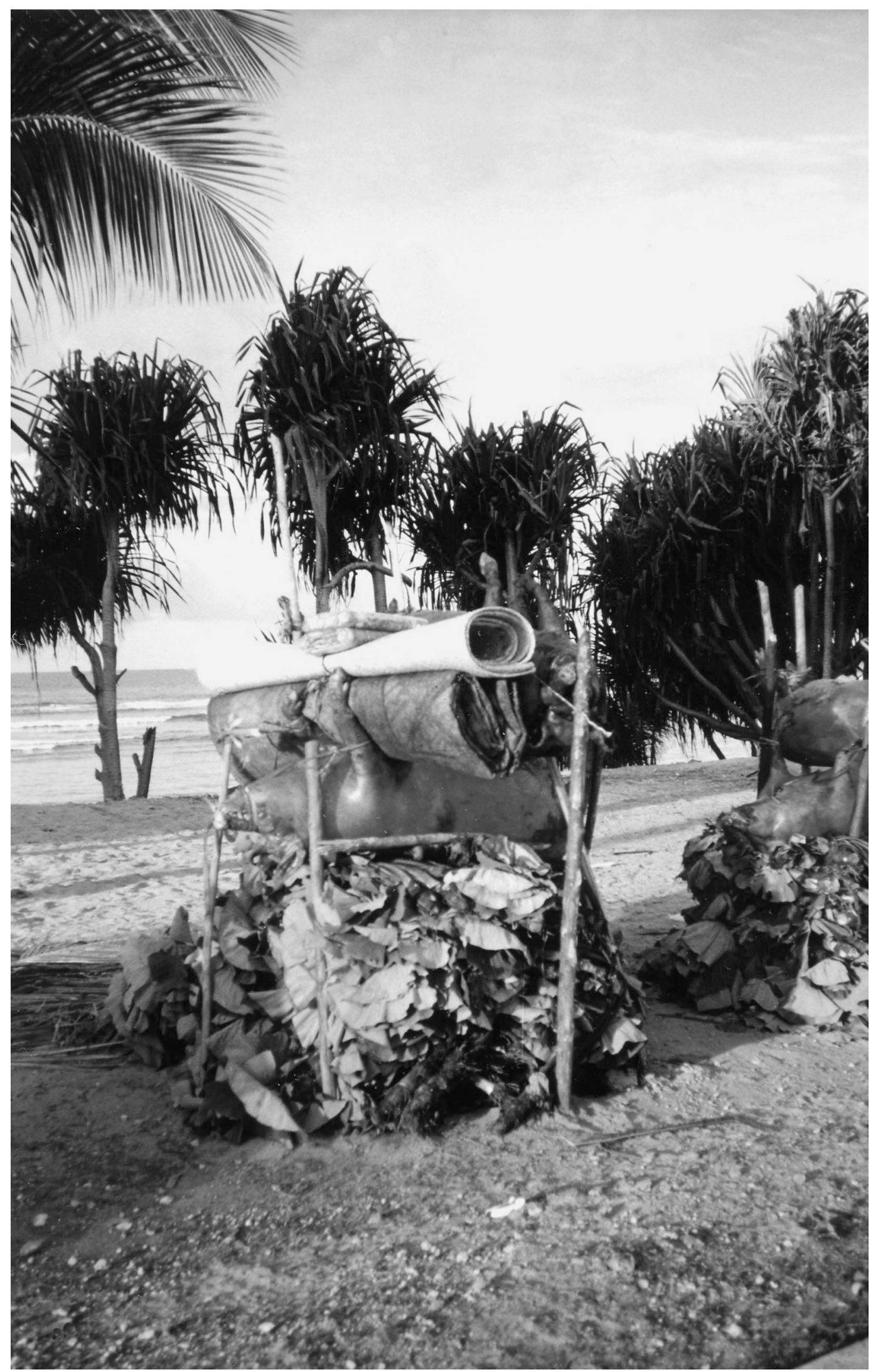

Рното 1. - L'un des cinq 'umu mata, les grandes « colonnes » de nourriture et de richesses donnés par les grands chefs et les élus à l'occasion du katoaga d'Ono (cliché Adriano Favole). 
attribuée en grande partie à Soane Masei (dit « Tāpili »), un jeune homme d'Ono avec qui j'ai tissé une amitié étroite et auquel me lie un rapport de profonde « empathie ». C'est lui qui, un soir, a introduit le sujet du fakamisimisi tout en exprimant la profonde satisfaction qu'il a ressentie en entendant réciter le texte. Ouvrier chargé du bitumage de la route, grand passionné du travail dans les jardins où, comme la plupart des Futuniens, il cultive du taro, de l'igname, des bananes, du manioc et du tabac, Soane commente, lors de cette soirée et des soirées suivantes, chaque strophe et chaque terme du texte. En fin d'après-midi, après son travail, Soane m'accompagne dans les champs pour me montrer directement les thèmes célébrés dans le fakamisimisi.

«Écouter le fakamisimisi me récompense de tous les efforts que j'ai fournis pour le katoaga. C'est en regardant le katoaga disposé sur le mala'e que tu "vois" notre coutume. C'est en écoutant le fakamisimisi que tu peux "ressentir" la coutume futunienne. » (Entretien du 10 octobre 1997)

Le fakamisimisi, comme j'ai eu ensuite l'occasion de le vérifier à travers l'enregistrement de versions du même texte réalisées par le regretté Kalepo Nau (village d'Ono) et Toviko Tukumuli (Kolia) - sans toutefois pouvoir assister à leur mise en scène - n'est pas une composition fixe. La tradition fournit un canevas et propose une série d'expressions et de stratégies rhétoriques que l'orateur modifie en fonction de ses goûts personnels et surtout des produits qui sont disposés sur le mala'e. Une règle générale concernant ces compositions - et qui n'est d'ailleurs pas toujours respectée - prévoit l'interdiction de prononcer directement le nom des produits pour lesquels l'orateur compose une strophe de remerciement. C'est en partie grâce à cette règle que naît le charme littéraire de ce texte, riche en figures de style telles que métaphores, métonymies, hyperboles, etc. En remerciant les habitants du village pour la préparation du katoaga, l'orateur décrit d'une par, leurs activités quotidiennes comme s'il est en train d'y assister et d'autre part le décor environnemental au sein duquel celles-ci s'effectuent. Le fakamisimisi est une représentation idéalisée et "poétique » du rapport entre les Futuniens et l'environnement dans lequel ils vivent, une expression artistique de leur travail dans les jardins ${ }^{11}$. En lui se mêlent les sons, les parfums, les couleurs et les émotions des horticulteurs de cette petite communauté de la Polynésie occidentale.
La version du Sa'atula est également très appréciée pour son caractère novateur. En effet, il trouve le moyen de mentionner, sous forme de vers, non seulement les produits traditionnels mais aussi les activités de ceux qui réalisent des travaux salariés, recevant ainsi des compliments et provoquant également des éclats de rire en raison du contenu imprévisible de la partie finale du texte.

\section{Une tentative d'interprétation}

Je vais tenter à présent de fournir certaines clés de lecture du texte. Pour des exigences de synthèse et de clarté, je divise mon commentaire, fondé en grande partie sur la traduction de Soane Masei, en paragraphes correspondant aux différentes strophes, tout en me référant à la version publiée en appendice.

\section{Exorde}

Après s'être levé du fale et avoir pris place à côté de la première rangée de biens formée par les plantes de kava, l'orateur prononce une sorte d'« appel» qui retrace la hiérarchie du village organisateur du katoaga. Les trois aliki sont suivis des anciens (kau matu'a) et des jeunes. Aliki, kau matu'a et tauleleka (les "jeunes déjà mariés ») sont les trois catégories statutaires d'hommes qui, lors des distribution de kava, forment l'alofi (le « cercle» du kava).

\section{Remerciement général}

Les chefs (définis par le Sa'atula comme « frères », faletautaina), sont remerciés pour avoir « organisé » (fa'ufa'u) et "dirigé » (lagi'aki) le katoaga. L'action des jeunes est, quant à elle, exprimée par le terme fakalogo, " obéir 》. Grâce à l'action conjointe des chefs, des anciens et des jeunes, l'île est «brune en raison des feuilles séchées ", une référence à la grande quantité de feuilles qui servent à la cuisson des aliments. Le mala'e, la place du village sur laquelle, les jours précédents, avait été disposée une nouvelle couche de corail, est « noir comme la nuit » en raison de l'énorme quantité de biens exhibés.

\section{Le kava}

C'est la première strophe consacrée à un produit exposé sur le mala'e, le plus important du point de vue hiérarchique, à savoir les racines de kava. Le Sa'atula remercie les anciens et les jeu-

11. À propos de jardins et de la gestion des espaces forestiers à Futuna, voir Anne Di Piazza (1993) et Hélène Guiot (2000). 


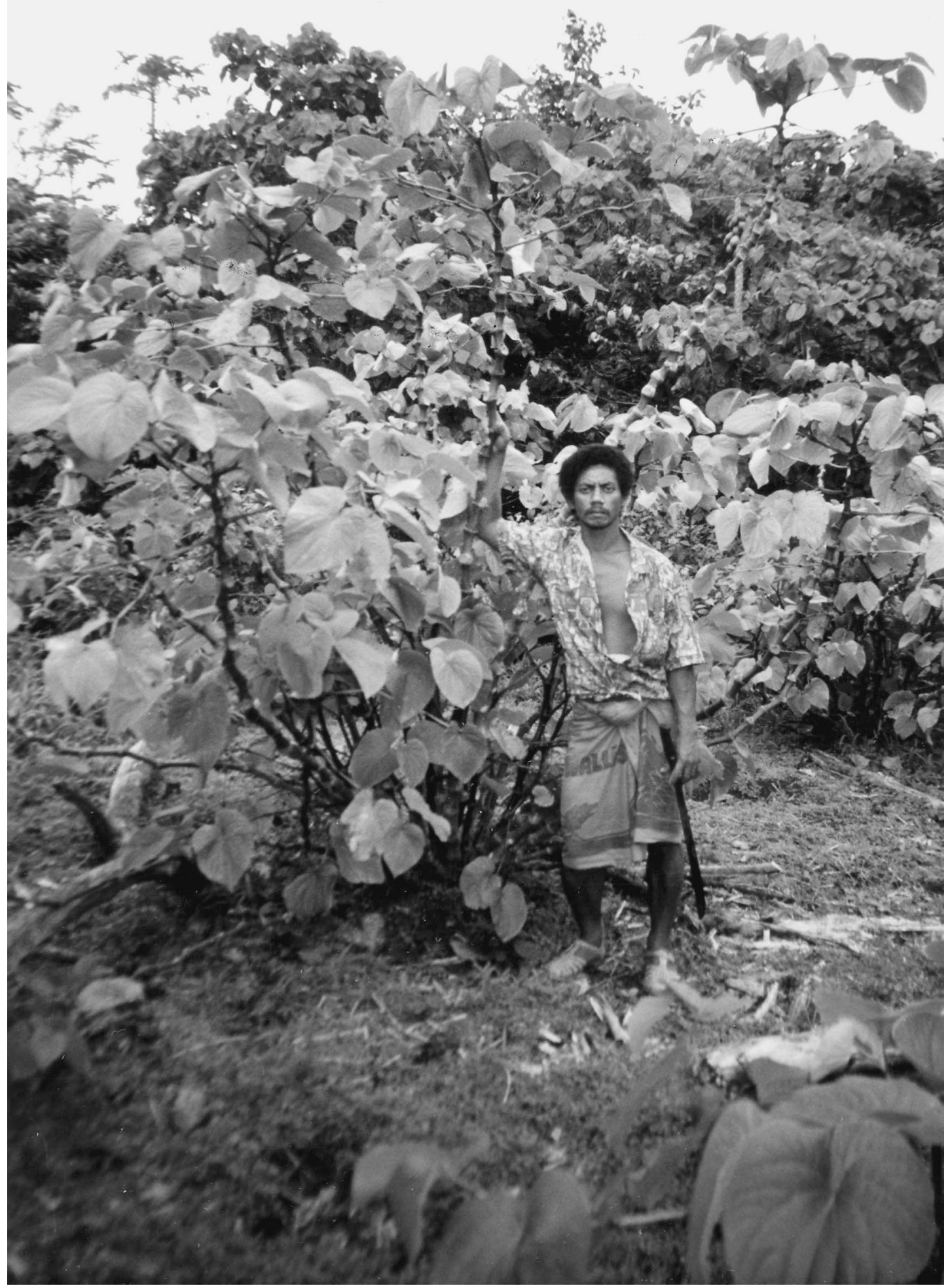

Рното 2. - Une grande plante de kava coupé pour la cérémonie, île d’Alofi (cliché Adriano Favole). 
nes pour avoir apporté de très « vieux » kava c'est-à-dire de grandes dimensions. L'idée de la " grandeur » est suggérée par la présence des fourmis (lo'ata) qui se mordent (fe'uti) entre les racines (tellement elles sont nombreuses!), par les lierres grimpants qui recouvrent désormais les plantes, par le fait que les branches se sont allongées au point de se courber et d'aller sous terre en formant de nouveaux rejetons ('ugamea) et enfin par l'hyperbole finale à travers laquelle les racines du kava sont comparées à celles des gigantesques arbres aoa (Ficus prolixa). Un " grand » pied de kava est appréciable non seulement parce qu'il permet de produire de remarquables quantités de boisson mais aussi parce que le goût de celle-ci sera plus « fort ».

\section{Le cochon}

Seconds par ordre d'importance derrière le kava, les grands cochons peints avec le rouge du curcuma trônent sur le mala'e. La strophe décrit les différentes activités qu'accomplissent les hommes pour les élever : le cassage des noix de coco et la préparation du manioc qui requièrent un nettoyage continuel de l'espace entourant les fale (muli fale; mu'a fale). C'est ce travail continu et difficile qui permet à présent l'exhibition de cochons aussi gros. Le remerciement pour leur grandeur est une nouvelle fois exprimé à travers l'utilisation de métaphores : le cochon est comparé à « celui qui ronfle » (sogulu) car les animaux les plus gros et vieux ronflent comme les êtres humains. L'expression qui suit - «la fleur de tiale s'est épanouie »- est une allusion aux incisives des cochons les plus vieux qui sortent de leur bouche. Lorsque l'animal atteint des dimensions exceptionnelles, ces mêmes dents se courbent comme « la fleur du pandanus ».

\section{Les paniers de nourriture}

L'image de l'«île enveloppée » par la fumée est encore une hyperbole qui se réfère aux grandes quantités de nourriture préparées et cuites dans les fours provoquant ainsi la diffusion de la fumée dans l'île entière. Les jeunes et les anciens sont remerciés pour s'être «brûlés» (vela), une allusion à la souffrance provoquée par le fait qu'ils se trouvent à proximité des fours lors de la préparation des aliments.

\section{L'igname}

Les ignames auxquelles se réfère cette strophe - sans les nommer directement - sont une composante importante d'un katoaga. L'expression « les nids sont remplis » réfère à la façon dont les ignames sont plantées, c'est-à-dire en enterrant une partie d'un tubercule et en la recouvrant d'un monticule de terre qui s'apparente à un nid renversé. La grandeur des ignames présentes sur le mala'e est exprimée à travers une comparaison avec les grands requins (tanifa) qui dorment (moe) au fond de la mer. Seules les grandes ignames produisent des rejetons qui poussent, enroulés, semblables aux murènes dissimulées dans les anfractuosités des rochers.

\section{Le taro sec}

Les différentes strophes suivent un schéma général : un remerciement collectif tout d'abord, puis la description du travail et enfin la constatation des brillants résultats auxquels il a donné lieu. Dans cette strophe est mentionné le taro sec - ou « taro de montagne »-planté sur les reliefs après que la forêt a été déboisée et brûlée et le terrain défriché. L'expression « avoir enlevé les toiles d'araignée » doit être comprise comme un compliment à l'égard de ceux qui sont passés les premiers sur un sentier et qui, par conséquent, ont commencé à travailler de bonne heure. Misikivi indique l'état du taro au moment où la récolte peut avoir lieu lorsque de nombreuses feuilles sont tombées. Le taro est "assoiffé » (pakupaku, terme souvent utilisé pour faire une allusion au désir sexuel) comme le montrent les feuilles sèches qui sont désormais tombées.

\section{Le taro d'eau}

La partie initiale de la strophe décrit les activités nécessaires à la culture du taro irrigué. Les fatumanu sont les petits rejetons qui poussent sur le tubercule désormais prêt à être ramassé. Ces derniers permettent de produire le faikai tutu, un aliment prestigieux (taro d'eau râpés et cuit dans le lait de coco) qui est offert au roi tout de suite après son intronisation. Tamolevai est une algue particulière dont la présence signale d'une part la récolte imminente du taro et d'autre part son abondance.

\section{Le kape}

Le kape (Alocasia macrorrhiza) pousse dans la forêt entre les racines des arbres. Dans ce cas également, la plante n'est pas nommée directement mais à travers une série de métaphores. $\mathrm{La}$ fleur du kape est comparée au bois appelé 'afa (Neonauclea forsteri) auquel elle ressemble par sa couleur et le parfum qu'il exhale. Lorsque ses feuilles tombent - une référence à un grand kape prêt à être ramassé - son tronc reste dépouillé comme le torse d'un homme nu. La comparaison 
avec le lali, le «tambour» futunien, est une hyperbole pour indiquer la grandeur des kape présents sur le mala'e. L'image suivante désignant les oiseaux qui utilisent le tronc rongé par les rats pour y construire leurs nids est également une hyperbole. L'abondance des kape produits par les hommes du village justifie un «gaspillage $»$ : ils servent de nourriture et de refuge pour les animaux!

\section{Les bananes}

Une fois encore, le fruit faisant l'objet du remerciement n'est pas nommé. Toutefois, la référence est explicite pour un Futunien : les tu'ulāosi sont les nouvelles plantes de bananier qui poussent tout autour du tronc principal et qu'il faut continuellement couper. Laulu est le terme spécifique désignant les feuilles de banane tandis que tu'amulikolo est la «fleur» rouge d'où provient le régime. Ce dernier est comparé aux fruits du falasola, une variété de pandanus dont les fleurs permettent la création de colliers. En effet, les fruits falasola, une fois arrivés à maturation, s'ouvrent " comme un régime de bananes ». La référence à la " chute» des falasola est une allusion aux bananes si abondantes qu'elles tombent d'elles-mêmes. La partie finale de la strophe exalte une nouvelle fois le thème de l'abondance. La stratégie rhétorique consiste en la description de ce qui se produit lorsque les régimes de bananes sont laissés sur les plantes, même s'ils sont désormais bien mûrs (une circonstance rare à Futuna!). Dans ce cas, des nuages d'insectes miomio'i manono, les petites mouches à fruits, entourent les bananes mûres tandis que les lézards en profitent pour monter et descendre le long de la plante (sōlia terme emprunté à l'anglais, signifiant littéralement « soldats » ou plutôt " marcher en rang comme des soldats ») et se gaver de ces mêmes moucherons. Les oiseaux miti (Aplonis tabuensis), avec leur cri strident caractéristique, se disputent eux aussi les fruits mûrs laissés sur les arbres par les hommes.

\section{Le tabac et les biens occidentaux}

Je réunis ces deux strophes dans la mesure où elles constituent un remerciement pour la production de biens récents d'origine occidentale : le tabac et l'argent. La strophe 11 décrit la culture du tabac qui est effectuée sur des terrains moins fertiles, là où affleurent des roches laviques. Il est demandé aux Futuniens qui exercent des travaux salariés d'offrir pour un katoaga de l'argent et des objets d'importation (viande en boîte, cigarettes, briquets, riz, biscuits, etc.). La strophe suivante les décrit, à travers une pointe d'ironie voilée, en train de " travailler » avec des stylos et de garder les clés des bureaux et les remercie pour leur intelligence (atamai) et leur ponctualité (tulikaki o le temi), deux qualités retenues comme indispensables pour exercer ga'oi papālagi, les «travaux des Blancs». L' "étendard» (manumanu) auquel la strophe fait référence est formé d'une centaine de pièces d'étoffe colorées : il est également comparé à l'arc-en-ciel (nиапиа). Dans un katoaga, ce dernier est hissé à proximité des 'umu mata, au-delà de la route. Ces deux strophes furent inventées de toutes pièces par Setefano Takaniko.

\section{Les biens féminins}

L'ordre hiérarchique des biens annoncé par le fakamisimisi place les biens féminins (koloa) en dernière position même si les nattes et les étoffes d'écorce étaient déjà disposées près des cochons qui occupaient les premières rangées du katoaga. Les femmes sont remerciées pour leur activité de femmes-fatogia. Les produits de leur travail sont évoqués par des gestes et des sons réalisés lors de ces mêmes activités. L'action consistant à « enlever les épines du pandanus », « le grincement» que produit le couteau sur ces mêmes feuilles afin de les assouplir ainsi que la division de ces dernières en de fines lamelles sont des moments caractéristiques de la réalisation des nattes. Quant à la production d'étoffes d'écorce et d'huiles parfumées - pour lesquelles sont utilisés les fruits ifiifi et les fleurs mosokoi (Cananga odorata) cités dans la strophe - ces activités sont typiquement féminines. Le ramassage et le raclage des racines matatuna avec lesquelles est produite la poudre du curcuma (ama) est une autre tâche accomplie par les femmes. Casser les noix de coco pour les cochons est, en revanche, un rôle purement masculin. L'expression déclamée en fin de strophe est un compliment adressé aux femmes pour s'être également chargées de tâches masculines !

\section{Conclusion}

Arrivé à la dernière rangée de biens sur le mala'e - les 'umu mata -, le Sa'atula énonce la dernière strophe. La « lutte » contre la forêt et la végétation sauvage qui croît rapidement pour « déchirer » (sae) la terre de cette dernière et la destiner à la production des ressources pour les êtres humains est la synthèse finale à travers laquelle est décrit le travail des hommes et des femmes. Cette activité permanente de déboisement pour obtenir une terre cultivable a rendu possible la réalisation du katoaga et a rendu 


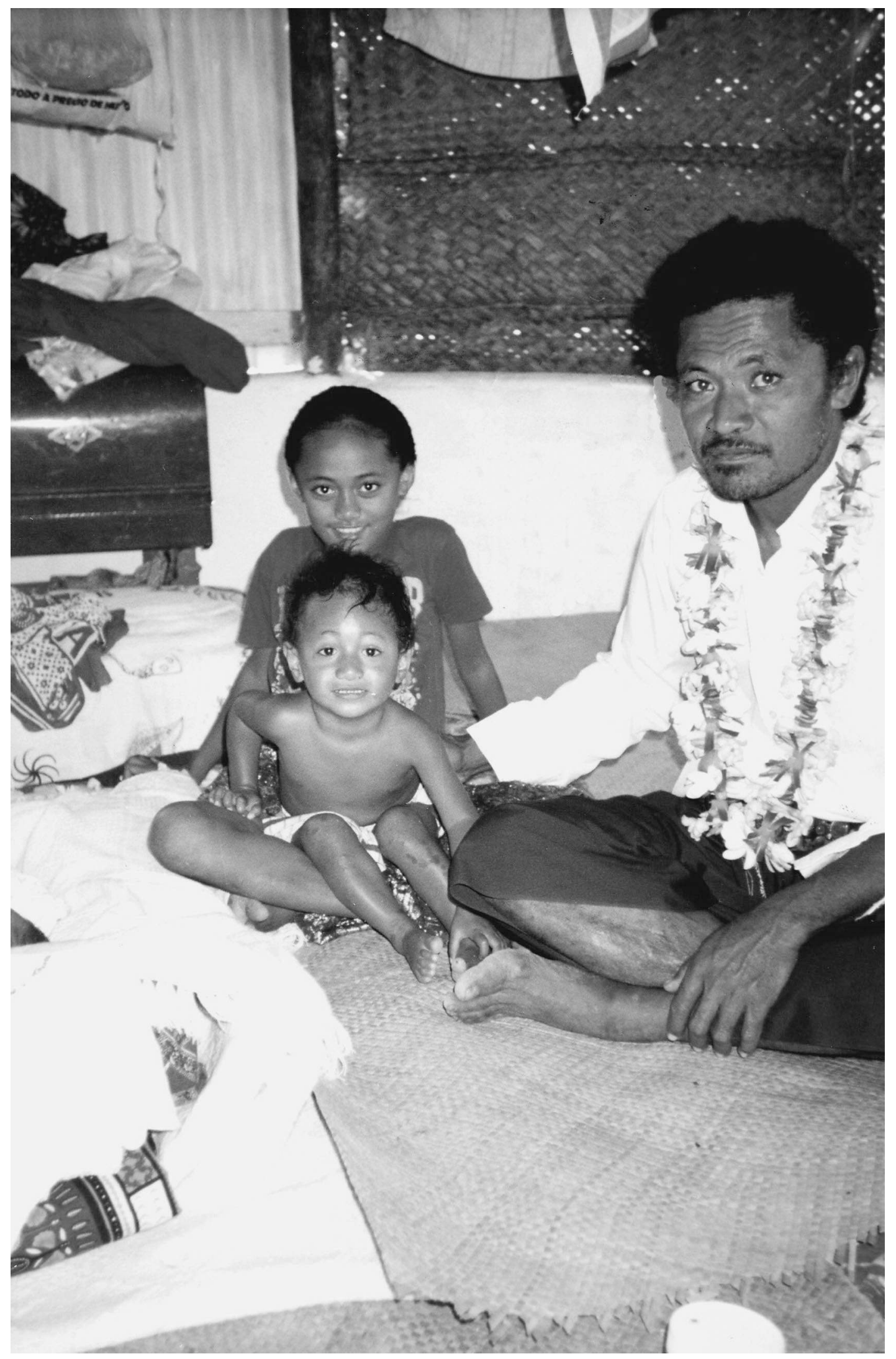

Рното 3. - Soane Masei avec deux des ses enfants dans son habitation à Ono (cliché Adriano Favole). 
«l'ensemble magnifique ». Le travail et l'effort de transformation de l'environnement permettent à l'île d'être manu'ia, "prospère" et «vitale ». Le fakamisimisi se termine par un remerciement à l'égard de Dieu.

\section{Esthétique de la coutume}

Les katoaga futuniens sont des événements qui ont une centralité précise dans la vie sociale, politique, économique et religieuse de l'île. Célébrations de la « citoyenneté » (fatogia), ces longues cérémonies fournissent aux chefs une occasion pour légitimer leur propre pouvoir. En effet, dans la représentation du katoaga, la société se présente comme un ensemble de catégories sociales liées entre elles par un rapport hiérarchique (aliki, kau matu'a, tauleleka, fafine). Sur le même modèle, les produits du travail sont eux aussi disposés de façon hiérarchique (les kava, les cochons, les paniers de nourriture, etc.). Les katoaga et les kava futuniens peuvent être définis de ce point de vue comme des rites " cosmopoétiques » (Favole, 2000a, 2000b) dans la mesure où ils aspirent à construire (poiêin) un « ordre » (cosmos) dans le monde des hommes et de la nature à travers la définition de catégories hiérarchiques ${ }^{12}$. Comme l'observe Patrick Kirch (1994a : 199), les katoaga fournissent également aux chefs une " arène politique » dans laquelle ces derniers peuvent se défier. En effet, les différents chefs de l'île ne rivalisent pas seulement à distance à travers la magnificence des katoaga organisés dans leurs villages respectifs. Le « défi » a lieu également au sein de chacune des cérémonies à travers les dons et les performances. Le katoaga du $1^{\text {er }}$ octobre 1997 dans le village d'Ono a été l'occasion d'un affrontement véritable entre le Sa'atula, grand chef du village de Mala'e et auteur du fakamisimisi, et le Tu'i Sa'avaka, grand chef de Kolia auquel a été confiée la déclamation d'un miō, récité aussitôt après. Les deux chefs s'étaient déjà affrontés à l'occasion de la nomination du nouveau Tu'i Agaifo, le roi d'Alo, lors du mois de juillet précédent. L'ancien Tu'i Sa'avaka fait partie des prétendants au titre tandis que Setefano Takaniko (qui n'est pas encore devenu aliki) est l'un de ses opposants. Lorsque Takaniko devient Sa'atula, au mois d'août de la même année, le Tu'i Sa'avaka menace de ne pas participer à la cérémonie d'investiture. Il s'y présente d'ailleurs avec plusieurs heures de retard au moment où l'on s'apprête à renvoyer l'élection. Le katoaga $\mathrm{du} 1^{\mathrm{er}}$ octobre représente la continuation et le point culminant du défi : tandis que le Sa'atula déclame le fakamisimisi de manière impeccable en éveillant l'attention et en recevant l'approbation du public, le Tu'i Sa'avaka ne se montre pas à la hauteur. En effet, il s'interrompt plusieurs fois, donnant ainsi l'impression d'avoir oublié les paroles. Sa performance est rejetée par de sonores éclats de rire et, quelques jours plus tard, de nombreux habitants du village disent que son comportement a couvert de honte le village de Kolia. La déclamation peu réussie du texte sanctionne d'ailleurs son déclin politique.

Les katoaga peuvent s'inscrire dans ces "politiques de la tradition" auxquelles l'anthropologie océaniste a consacré de nombreuses réflexions au cours des années quatrevingts-dix. Ils mettent en scène une représentation spectaculaire de la coutume considérée comme un élément de différenciation du monde extérieur et d'affirmation de l'identité locale. La référence au kastom et au passé ne doit pas être comprise au sens essentialiste comme s'il s'agit de proposer de nouveau une identité « originaire », " pure » et précoloniale. Les katoaga, comme il a été dit depuis le début, se célèbrent aujourd'hui pour les saints chrétiens patrons des villages : à ces occasions, la contribution en argent et en biens qui est octroyée à la mission catholique est d'une grande importance.

Du point de vue des relations sociales, les katoaga, comme le remarquait déjà Raymond Firth pour Tikopia (1965: chap. 6), sont une incitation à la production et au travail ainsi qu'une occasion de rencontre et de coopération entre les membres des groupes de parenté les plus étendus (kūtuga o fāmili à Futuna) qui vivent çà et là dans différentes parties de l'île. Du point de vue économique, il faut observer que ces cérémonies exercent aujourd'hui un rôle important dans la redistribution des ressources monétaires. Ainsi, il est demandé aux Futuniens qui effectuent des travaux salariés d'offrir de généreuses contributions financières pour la fête ${ }^{13}$. Ces sommes d'argent sont ensuite offertes en partie à la mission catholique, en partie aux coopératives de village et sont également utilisées pour les travaux d'intérêt collectif. Au cours de ces dernières années, le fait que le nombre de katoaga

12. La pratique du pouvoir, comme je l'ai montré ailleurs (Favole, 2000b), dément souvent cette représentation hiérarchique de la société offerte par les rituels, en laissant émerger les aspects conflictuels, égalitaires et, en définitive, "démocratiques » (Favole, 2005) de la vie sociale de cette communauté polynésienne.

13. Dans ce cas précis, chaque homme-fatogia exerçant un travail salarié se devait de donner $20000 \mathrm{~F} \mathrm{CFP.}$ 
ainsi que les ressources exhibées aient particulièrement augmenté ${ }^{14}$ semblerait pouvoir s'expliquer par la tentative d'éviter que dans une économie telle que celle de Wallis-et-Futuna dans laquelle seule une partie des groupes familiaux peut compter sur des emplois salariés et dans laquelle le poids des mandats envoyés sur l'île par les émigrés augmente peu à peu - se créent de fortes inégalités économiques $^{15}$. Les katoaga constituent aujourd'hui une réponse «traditionnelle » à un problème contemporain, la présence locale de salariés et d'une forte communauté externe d'émigrés qui envoient des ressources considérables sur l'île, créant ainsi une fracture importante entre les familles pouvant compter sur des rentrées de nature monétaire et la majorité des groupes familiaux qui continuent à pratiquer une agriculture de subsistance.

Un autre aspect relatif à ces cérémonies, mis précisément en évidence par le fakamisimisi et sur lequel je souhaite concentrer mon attention dans la partie finale de cet essai, relève de ce nous pourrions définir l'« esthétique de la coutume ». En suivant la préparation et le déroulement d'un katoaga, d'un kava officiel ou encore la fabrication de produits traditionnels comme les étoffes d'écorce (tapa et siapo), le curcuma (ama) et l'huile de coco (lolo) - des produits qui appartiennent tous au circuit d'échanges à l'occasion des katoaga et qui sont célébrés de façon solennelle par le fakamisimisi - il est difficile de se soustraire à l'impression qu'il existe, parmi les objectifs de leur réalisation, la recherche d'une sorte d' "appréhension esthétique » de la part des participants. Le spectacle offert par la place du village recouverte de cochons rougis éclairés par les premières et pâles lumières du soleil, de paniers en feuilles de coco tressées, d'étoffes d'écorce décorées d'encres locales et de l'étendard d'étoffes s'élevant près de la plage, n'est pas seulement considéré comme étant de toute beauté par des observateurs externes. En effet, après avoir disposé le katoaga, les hommes et les femmes se sont assis pour contempler cette image, représentation artistique de leurs activités quotidiennes. À travers le fakamisimisi, le chef en offrit ensuite une représentation textuelle solennelle.

Lors de ces mêmes occasions, le soin particulier que les Futuniens mettent à préparer et cuire les aliments, embellir les lieux servant de décor aux célébrations et orner leur corps - colliers et couronnes de fleurs, tatouages, jupettes en feuilles, huile parfumée pour la peau, tressage des cheveux, etc. - suggère l'idée qu'une recherche du « beau et du sublime » est à la base de ces rituels. La capacité d'un katoaga à intéresser, à mobiliser des ressources et du travail et à inciter à redistribuer les richesses naît également de sa nature d' "expérience du beau», expression esthétique mise à la disposition de tous et non pas seulement d'une catégorie sociale en particulier, telle une sorte d'ennoblissement artistique du travail quotidien. Il n'est pas improbable qu'à la base des «politiques de la tradition» qui, dans la situation post-coloniale, caractérisent de nombreuses sociétés du Pacifique, subsiste également le désir de récupérer des expériences esthétiques - qui ne sont pas nécessairement liées aux productions d'objets d' " art » affranchis de l'activité quotidienne - qui risquent de disparaître suite à l'impact de la mondialisation et des marchandises standardisées.

\section{ANNEXE}

\section{Exorde}

\author{
Kua 'au Tu'i Asoa \\ kua' 'au Fainuvele \\ kua 'au Ma'nifa \\ kua 'au le kau matu'a \\ kua' 'au tauleleka.
}

\author{
Voilà, le Tu'i Asoa arrive \\ le Fainuvele arrive \\ le Ma'uifa arrive \\ les anciens arrivent \\ les jeunes mariés arrivent.
}

14. Dans le katoaga d'Ono, un ensemble de cent soixante cochons a été exhibé.

15. Sur le rapport entre économie de marché et économie locale, se référer aux travaux de Paul van der Grijp (2002 et dans ce volume). Trois représentants du village d'Ono qui résident à Nouméa ont participé à la cérémonie du $1^{\text {er }}$ octobre et fait don de $140000 \mathrm{~F} \mathrm{CFP}$ 


\title{
Remerciement général
}

kia koutou faletautaina

ki le fa'ufa'u mo le lagi'aki;

tokoi a le kau matu'a

tali fakalogo a tauleleka

kua fulumalie le aso

kua mea le fenua

kua tu'u fakapo'uli mala'e.
Ku kau fakamālō atu

Je vous remercie

frères chefs,

pour votre œuvre d'organisation et de direction.

Grâce à l'aide des anciens

et à l'obéissance des jeunes,

cette journée est magnifique.

L'île est devenue brune,

La place du village est noire comme la nuit.

\section{Le kava}

\author{
Ku kau fakamālō atu \\ ki matu'a mo tauleleka \\ tafitafi o le lalo mei \\ sunuki o le fatikata \\ kua fe'uti le lo'ata \\ kua funa le kava \\ kua mafuta le 'ugamea \\ kua visi le aoa.
}

Ku kau fakamālō atu

ki matu'a mo tauleleka

tāpō le lokiloki

taupau o le muli fale

teuteu'i le mu'a fale

tege o le niu matu'u

palasi o le manioka

kua toka le sogulu

kua sē le tiale

$k u(a)$ matule sigano.

Ku kau fakamālō atu

ki le vela a matu'a mo tauleleka

i le tali o le lagi'aki

kua kofu le fenua

kua sikumolomolo le launiu

kua gini le laumei.

\author{
Je vous remercie \\ anciens et jeunes, \\ pour avoir déboisé et défriché [la terre] sous les arbres \\ à pain, \\ pour avoir planté les boutures ; \\ les fourmis se mordent entre elles, \\ le kava est couvert de plantes grimpantes, \\ de nouveaux rejetons ont éclaté, \\ les racines des arbres aoa sont désormais enchevêtrées.
}

\section{Le cochon}

\section{Les paniers de nourriture}

\author{
Je vous remercie \\ anciens et jeunes, \\ pour avoir supporté la chaleur, \\ pour avoir répondu aux ordres ; \\ à présent, l'île est enveloppée dans la fumée, \\ les feuilles de cocotiers sont enchevêtrées en sikumolo- \\ molo, \\ les feuilles de l'arbre à pain sont fanées.
}

\section{L'igname}

\author{
Ku kau fakamālō atu \\ ki matu'a mo tauleleka \\ i le tulei o le kala'apusi \\ tu'uti o le faliava \\ fakagini o le pulopulou;
}


kua tu'u le ōfaga

fakamapāo le matākoso

fulusi o le kele

kua moe le tanifa

mamiomio tāpea. à présent, les « nids » sont remplis,

la terre « explose » sous la force du bâton à fouir, elle se retourne ;

les grands requins dorment, les murènes s'entortillent.

\section{Le taro sec}

\author{
Ku kau fakamālō atu \\ ki matu'a mo tauleleka \\ i le 'ofo o le ma'uli \\ fakatapa a le 'aselo \\ i le tā o le kaleveleve \\ fakasali o le kakava \\ tule'i o le pala vao \\ fakamapā o le aka'i la'akau \\ kua manogi le pua felo \\ kua misikivi talo pakupaku.
}

\author{
Je vous remercie \\ anciens et jeunes, \\ le matin, la vie s'anime et \\ suite à la prière à l'ange gardien, \\ vous avez enlevé les toiles d'araignée, \\ la sueur a coulé sur votre front ; \\ les arbres de la forêt tombent, \\ leurs racines « explosent »: \\ la fleur jaune embaume, \\ le taro « assoiffé » a perdu ses feuilles.
}

\title{
Le taro d'eau
}

Ku kau fakamālō atu ki matu'a mo tauleleka i le taupau o le matapuna tafitafi o le mata'i lava fetuku'aki o le pela kua fufu kua ino le vai le laku o le limu kua mafuta le fatumanu kua muligāmanu le tamolevai.

\author{
Ku kau fakamālō atu \\ ki matu'a mo tauleleka \\ i le tā o le sasau \\ kapusaki o le mapu \\ fakalele o le manu; \\ Fakatagi o le vao matu'a \\ fakalaka o le koso \\ kua sē le 'afa \\ kua maluku le lau \\ kua tu'u fakatekateka le tagata \\ kua tã lali a kimoa \\ fai nofo'aga a manu.
}

\section{Le kape}

\author{
Je vous remercie \\ anciens et jeunes, \\ pour vous être occupés des sources, \\ pour avoir nettoyé les canalisations d'eau, \\ pour avoir nivelé la boue : l'eau est trouble, \\ la terre se dessèche, \\ les algues sont rejetées sur les bords. \\ À présent, les fatumanu poussent, \\ l'algue tamolevai est désormais apparue.
}

Je vous remercie

anciens et jeunes,

la rosée vous frappe,

[votre] respiration haletante et sifflante

fait s'envoler les oiseaux.

La vieille forêt pleure,

le bâton passe à travers les racines :

l'afa s'est épanoui,

les feuilles tombent

et le tronc reste là debout comme un homme nu, les rats frappent un tambour,

les oiseaux y construisent leurs nids.

\section{Les bananes}

Ku kau fakamālō atu

ki matu'a mo tauleleka

taupau o le tu'ulāosi

tu'uti o le laulu

tule'i o le tu'amulikolo ;

ku leleu o le kafika

kua maluku le falasola

kua mafuta le miomio'i manono

kua sōlia a pili

kua kī masaesae o miti.
Je vous remercie

anciens et jeunes,

pour vous être occupés des nouveaux rejetons,

pour avoir coupé les feuilles sèches,

pour avoir tranché la fleur rouge :

à présent, les fruits kafika mûrissent,

les fruits du falasola tombent,

les insectes miomio'i manono volent tout autour,

les lézards pili montent en file indienne,

les oiseaux miti poussent de grands cris. 


\section{Le tabac}

\author{
Ku kau fakamālō atu \\ ki matu'a mo tauleleka \\ i le tatafi o le fuga tule \\ muluki o le fakaili \\ fakalaka o le mata'i sele \\ fakamapā o le maikao \\ kua lasi le tapaka \\ kua kofu fuli a paipa.
}

\author{
Je vous remercie \\ anciens et jeunes, \\ pour avoir nettoyé les terres couvertes de roches volca- \\ niques, là en haut, \\ pour avoir semé en frottant les graines entre vos mains, \\ pour la lame de couteau qui a franchi les cailloux, \\ pour avoir enterré [les petites plantes] avec vos doigts, \\ à présent, le tabac a poussé \\ toutes les pipes fument.
}

\section{Les biens occidentaux}

\author{
Ku kau fakamālō atu \\ ki matu'a mo tauleleka \\ i le poto mo le atamai \\ tulikaki o le temi \\ takai o le peni \\ tāpō o le kalavi \\ kua lasi a koloa papālagi \\ kua sā le nuanua \\ kиa tu'u manuтапи o le fenиa.
}

\author{
Je vous remercie \\ anciens et jeunes, \\ pour votre instruction et votre intelligence, \\ pour votre ponctualité, \\ vous avez travaillé avec des stylos, \\ vous avez surveillé les clés : \\ les richesses occidentales sont désormais abondantes, \\ l'arc-en-ciel est apparu \\ le drapeau de notre terre est hissé.
}

\section{Les biens féminins}

\author{
Ku kau fakamālō atu \\ kia koutou kau fafine matu'a \\ ti mo koutou kau fafine makeke \\ i le tali o le lagi'aki \\ mo le tali o le fatogia ; \\ fakamafiti o le 'autala \\ fakatagi o le sa'alo \\ fakamapā o le maikao; \\ fakatagi o le tutua \\ sokosoko o le laututu \\ fakamalū o le ifiifi \\ filio'i le mosokoi \\ fakama'opo o le matatuna \\ fetuku'aki o le maikao \\ fakatagi o le tuvai \\ kua ma'ua le tagata \\ kua kātoa fuli koloa o Futuna.
}

\section{Je vous remercie femmes âgées et jeunes femmes, pour avoir accepté les dispositions et avoir accepté le fatogia.}

[Je vous remercie] pour avoir retiré les épines des feuilles de pandanus, pour le grincement du couteau, pour avoir coupé les feuilles en lamelles ; pour avoir frappé l'écorce sur l'enclume en bois, pour avoir fait adhérer les bandes les unes aux autres, pour avoir recueilli les fruits ififi et avoir séparé les pétales des fleurs mosokoi;

[je vous remercie] pour avoir recueilli les racines matatuna et en avoir séparé les appendices ; en raclant les noix de coco, vous avez effectué les rôles des hommes : désormais, les richesses de Futuna sont vraiment toutes présentes.

\section{Conclusion}

Ku kau fakamālō atu

i le mākava a matu'a mo tauleleka i le solo'i o le 'ulufenua

sae o le kele

kua toka le teuteu

kua fulumalie le nofo

kua sā le mōliga

kua manu'ia le fenua

ti kua agatonu ki le Atua.
Je vous remercie

anciens et jeunes pour avoir préparé le kava, pour avoir déboisé la forêt éloignée des villages, pour lui avoir déchiré sa terre : tous les biens sont réunis ici devant nous, tout est magnifique : les dons sont exhibés, la terre est manu'ia, Rendons grâce à Dieu. 


\section{BIBLIOGRAPHIE}

ANGLEVIEL Frédéric, 1994. Les missions à Wallis-etFutuna au XIX ${ }^{\mathrm{e}}$ siècle, Bordeaux, CRET, coll. Îles et archipels 18.

Brosse Charles (de), 1756. Histoire des navigations aux terres australes, Paris, Durand.

Burrows Edwin. G., 1936. Ethnology of Futuna, Honolulu, Bernice P. Bishop Museum Bulletin 138.

Chanel Pierre,1960. Écrits de S. Pierre Chanel, établis, présentés et annotés par Claude Rozier, Rome, Éditions des pères maristes.

Di Piazza Anne, 1993. Les jardins enfouis de Futuna. Une ethno-archéologie de l'horticulture, Journal de la Société des Océanistes 97, pp. 151-162.

Di Piazza Anne, Daniel Frimigacci et Muni KeleTAONA, 1991. Hommes au four. Cuisine de Futuna, Nouméa, Éditions d'Art calédoniennes.

DouAIRE-MARSAUdON Françoise, 1998. Les premiers fruits. Parenté, identité et pouvoirs en Polynésie occidentale (Tonga, Wallis-et-Futuna), Paris, Éditions du CNRS.

FAvole Adriano, 2000a. La palma del potere. I capi e la costruzione della società a Futuna (Polinesia occidentale), Turin, Il Segnalibro.

_, 2000b. La royauté oscillante. Ethnographie et histoire de la cérémonie d'investiture du Tu'i Agaifo d'Alo (Futuna), Journal de la Société des Océanistes 111, pp. 195-218.

_, 2005. Une polyarchie polynésienne. L'organisation politique de Futuna et le débat sur la démocratie dans le Pacifique-sud, communication présentée au CREDO de Marseille (29/04/2005), n.p.

FIRTH Raymond, 1965. Primitive Polynesian economy, London, Routledge.
FrimigaCCI Daniel, 1990. Aux temps de la terre noire. Ethnoarchéologie des îles Futuna et Alofi, Paris, Peeters.

Frimigacci Daniel, Muni Keletaona, Claire Moyse-Faurie et Bernard Vienne, i995. Ko le fonu tu'a limulimua. La tortue au dos moussu. Textes de tradition orale de Futuna, Paris, Peeters, coll. Langues et cultures du Pacifique 11.

Grezel Isidore, 1878, Dictionnaire futunien-français avec notes grammaticales, Paris, Maisonneuve et $\mathrm{C}^{\mathrm{ie}}$.

GuIOT Hélène, 2000. Gestion traditionnelle des espaces forestiers à Futuna (Polynésie occidentale) : contenu idéel et pratiques associées, Journal de la Société des Océanistes 110, pp. 19-33.

KIRCH Patrick, 1994a. The wet and the dry. Irrigation and agricultural intensification in Polynesia, Chicago, The University of Chicago Press.

- 1994b. The pre-Christian ritual cycle of Futuna, Western Polynesia, Journal of the Polynesian Society 103, pp. 255-298.

Moyse-FAurie Claire,1993. Dictionnaire futunienfrançais, avec index français-futunien, Paris, Peeters, coll. Langues et cultures du Pacifique 8.

PANOFF Michel, 1970. La terre et l'organisation social en Polynésie, Paris, Payot.

Pechberty Dominique, I 998. Le katoaga, Journal de la Société des Océanistes 106, pp. 75-79.

SERvant Louis C., 1996. Écrits de Louis Catherin Servant, Paris, Pierre Téqui.

VAN DER GRIJP Paul, 2002. Selling is poverty, buying a shame: representations of work, effective leadership and market failures on Wallis, Oceania 73, pp. 17-34. 